\title{
P 102 VARIATIONS IN METHADONE PRESCRIBING AND OUTCOMES - A SERVICE EVALUATION IN SHEFFIELD AND CHESTERFIELD
}

Anne-Marie Bourke, ${ }^{1}$ Rebecca Theobald, ${ }^{1}$ Sam H Ahmedzai ${ }^{1,2}$. 'Sheffield Teaching Hospitals NHS Foundation Trust, Sheffield, England; ${ }^{2}$ The University of Sheffield, Sheffield, England

\subsection{6/bmjspcare-2014-000654.143}

Background Methadone is a synthetic opioid used in refractory pain. There is increased awareness of methadone related adverse events including respiratory depression and QTc prolongation. In our region several methods exist for methadone initiation, falling into two categories: switching one opioid to methadone (stop and go - SAG) and adding methadone to another opioid (addition - ADD).

Aims To characterise how methadone is being used locally. To quantify the nature and frequency of methadone induced side effects.

Methods All patients prescribed methadone by the palliative care teams in Sheffield and Chesterfield over an eight month period were included. Data were prospectively collected on diagnoses, indication(s) for methadone, method of conversion, ECG recording, pain scores and side effects.

Results 25 patients were prescribed methadone, all as inpatients. 20 patients had malignancy, the remainder chronic pain. Methadone was chosen in all cases due to persistent pain and/or medication side effects. A SAG method was used for $72 \%$ and ADD for $28 \%$. The median daily oral morphine equivalent dose prior to initiation was $240 \mathrm{mg}$ (range $50 \mathrm{mg}-1000 \mathrm{mg}$ ). 92\% had an ECG prior to commencing methadone.

Following titration, the median methadone dose was $10 \mathrm{mg}$ $\mathrm{BD}$ (range $4 \mathrm{mg}-40 \mathrm{mg} \mathrm{BD}$ ). Average pain scores reduced from $7 / 10$ to $3 / 10$ in both groups.

$28 \%$ reported side effects of sedation, nausea, confusion or hallucinations. 2 patients required naloxone and 3 developed QTc prolongation, all in the SAG group.

$72 \%$ were discharged home, $8 \%$ were transferred to another hospital and $20 \%$ died. None of the deaths were attributable to methadone.

Conclusion Locally, methadone is prescribed for patients with chronic pain as well as those with malignancy. Methadone results in clinically meaningful pain relief regardless of method of initiation. A SAG method is used most frequently. All five clinically significant adverse events were reported in the SAG group. 